\title{
VERIFICATION OF A DYNAMIC KNEE SIMULATOR COMPUTATIONAL MODEL
}

\author{
Amber N Reeve ${ }^{1}$, Mike A Strickland ${ }^{2}$, Lorin P Maletsky ${ }^{1}$, Mark Taylor ${ }^{2}$ \\ ${ }^{1}$ Department of Mechanical Engineering University of Kansas Lawrence, Kansas \\ ${ }^{2}$ School of Engineering Science University of Southampton Highfield Southampton, SO17 1BJ, UK
}

\section{INTRODUCTION}

Dynamic knee simulators aim to reproduce prescribed physiological loading and motions of the knee. The natural knee achieves stability through a complex interaction of the neuro-musculoskeletal system; thus, a knee simulator also requires a sophisticated control system to replicate human motion. Guess and Maletsky [1] developed a computational model to predict the required simulator inputs to produce the desired knee loading for dynamic activities on the Kansas Knee Simulator (KKS). The model built demonstrated conceptually that multi-body dynamics models could be used to simulate the KKS. However, as desired loading profiles became more complex, key limitations were discovered in the model; such as the model controller limited to a single axis under feedback control, no out-of-plane loading, not accounting for dynamic joint friction or damping of the actuators, and an inability of the model to flex pass $80^{\circ}$ degrees of knee flexion. Thus, there was a need for a new computational model to overcome the limitations and to provide a more robust and complete comparison to the KKS. The new computational model will allow better utilization of the KKS capabilities for future cadaveric and prosthetic testing. This work outlines the sagittalplane validation of the new computational model.

\section{MATERIALS AND METHODS}

Kansas Knee Simulator (KKS): The Kansas Knee Simulator [2] (Figure 1) is a five-axis servo-hydraulic machine capable of simulating dynamic loading activities on either cadaveric knees or total knee prostheses in test fixtures. The KKS has five axes of control: quadriceps, vertical load, medial-lateral, vertical torque, and ankle flexion. The KKS a five-channel PID controller (Instron 8800 Controller, Instron, Norwood, MA) with full cross compensation with the ability to operate any of the five axes in force or displacement control. The KKS does not control the kinematics of knee; the kinematics are a result of the forces generated by each axis and the constraint at the knee from geometry and structures. Generally, the quadriceps axis drives the hip flexion position and the other actuators are in load control to apply dynamic loading to the hip and ankle.

Computational Model of KKS: A computational rigid multi-body dynamics model has been developed in MSC.ADAMS (MSC Software Corporation, Santa Ana, CA), emulating the input loads, constraints, and degrees of freedom of the actual KKS (Figure 2). The complete simulator model includes over 30 parts to capture all inertial and dynamic effects; for example, it explicitly includes the actuators, with moving actuator rods, and individually modelled linkages, in order to include the additional damping effects and friction at the linkages. The control aspects of the KKS model are co-simulated in MATLAB/Simulink (The MathWorks, Inc, Natick, MA) to allow for more flexibility in methods of control. The control scheme was designed to implement basic PID feedback control and channel cross coupling for the five controllable axes. Switching the different feedback channels allows the user to toggle between force and displacement control of each axis. The new GUI-driven controller allows the user to easily monitor the controller behaviour in run-time and quickly visualize the internal signal routing of the axes. The model controller was tuned in a similar fashion as the physical system, by varying sine waves on cross-talking axes.

Model Verification: To verify that the model accurately represented the physical rig and its behaviour (forces generated by the actuators and the loading across the knee joint), sine wave profiles were constructed and ran on the KKS. For sagittal plane validation, sine waves were inputted on the quadriceps actuator (5-30 deg at $0.1 \mathrm{~Hz})$, vertical actuator $(37.5-187.5 \mathrm{~N}$ at $0.33 \mathrm{~Hz})$, and ankle actuator $(0-125 \mathrm{~N}$ at $0.5 \mathrm{~Hz}$ ). For all the tests, the quadriceps axis controlled the hip flexion angle and the other axes were in force-control. Comparisons were made between the feedback of the KKS and the Adams model for each axis and between the predicted loads and experimental loads across the joint, measured by a tri-axial load cell (JR3, Inc., Woodland, CA) mounted under the tibial tray. Differences between the model and the rig were calculated.

Profile Generation and Verification: An important function of the model is to predict the inputs required to control the axes of the KKS to generate desired loading at the knee. An equal stance squat profile was generated that attempted to maintain a constant compressive load across the joint. The hip flexion angle ranged from $5^{\circ}$ to $40^{\circ} \mathrm{deg}$ at $0.1 \mathrm{~Hz}$, and the compressive load was held at $500 \mathrm{~N}$. After the predicted input loads were generated from the model, the profile was run on a prosthetic knee in a test fixture. A tri-axial load cell mounted under the tibial tray measured the loading across the joint. The experimental joint 
loading was compared with the predicted loads from the Adams model. Differences between the model and the rig were calculated for the forces in the sagittal plane.

\section{RESULTS}

The root-mean-square (RMS) deviations between the model predicted loading and the measured loading of the rig for the sagittal sine wave profile were 188, 57, and $204 \mathrm{~N}$, for quadriceps load, anterior-posterior joint load, and joint axial compressive load, respectively (Figure 3). As a percentage of maximal loads, the RMS deviations were $8.4 \%$ for quadriceps load, $9.7 \%$ for anterior-posterior load, and $8.3 \%$ for axial compression. Similarly for the profile generation of a constant compressive load, the RMS deviations were 215,61 , and $239 \mathrm{~N}$ for quadriceps load, anterior-posterior joint load, and joint axial compressive load, respectively.

\section{DISCUSSION}

The KKS ADAMs model reasonably predicts the loading of the KKS physical machine and can reasonably produce profiles based on specified loading at the knee joint in the sagittal plane. The RMS deviations of the model are comparable to the values of Guess and Maletsky [1]. For the simulated profiles, the RMS deviation of the actuator loads and joint loads were within $10 \%$ of the maximum load. These loads are highly sensitive to alignment and interaction of the five axes. In general the KKS model under-predicts the actuator loads of the KKS. Future work on the KKS ADAMs model includes verification of out-of-plane loading, better characterization of damping, and an alternate scheme for controlling the five axes of the KKS.

The ability to create physiological profiles based on net knee loading for reproduction of the loading and improved accuracy of the model are desired capabilities of a computational model of the KKS. The new KKS ADAMs model better reflects the KKS geometry and inertia properties of the rig, includes 'deep flexion' capabilities (with quadriceps wrapping), and has a more sophisticated controller.

\section{ACKNOWLEDGEMENTS}

This work was supported by DePuy Orthopaedics, Inc., Warsaw, Indiana, a Johnson and Johnson company.

\section{REFERENCES}

1. Guess, T. M., and Maletsky, L. P., 2005, "Computational Modelling of a Dynamic Knee Simulator for Reproduction of Knee Loading," J Biomech Eng, 127, pp. 1216-1221.

2. Maletsky, L. P., and Hillberry, B. M., 2005, "Simulating Dynamic Activities Using a Five-Axis Knee Simulator," J Biomech Eng, 127, pp. 123133.

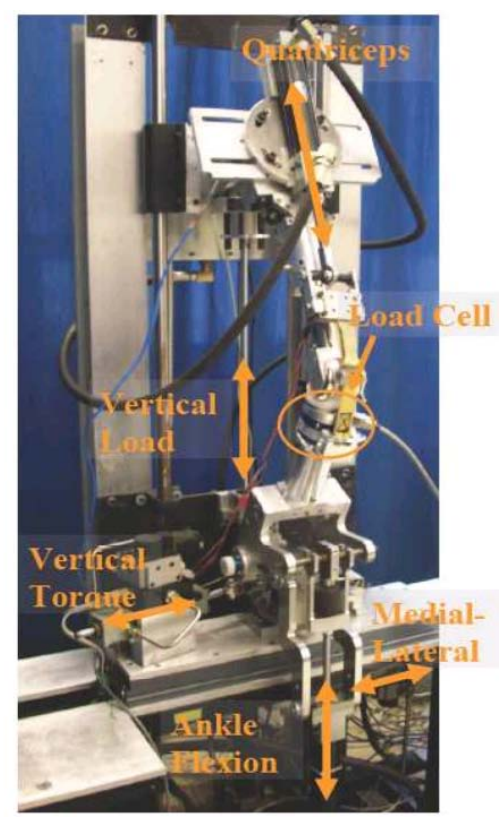

Figure 1. KKS with triaxial load cell mounted underneath the tibial tray. 


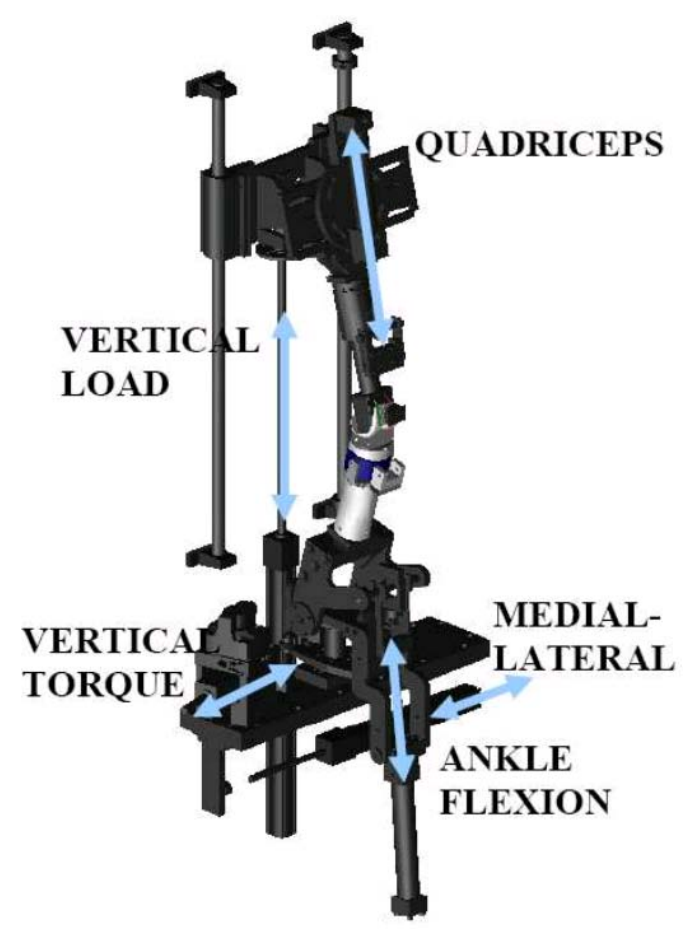

Figure 2. KKS computational model with triaxial load cell.
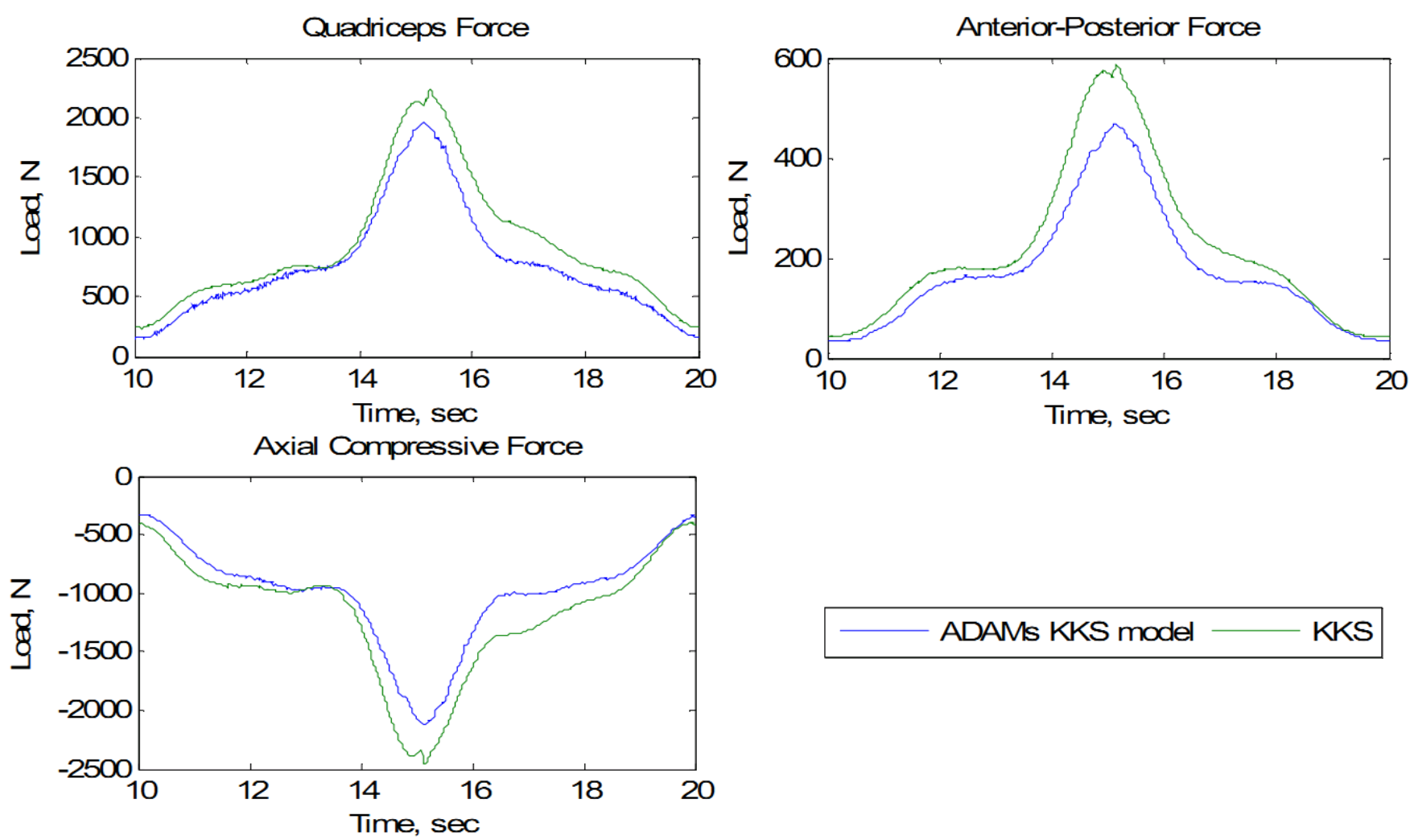

Figure 3. Predicted and measured loading for one cycle of the sagittal sine wave profile. 